Short Paper

\title{
Geeignete Bestäubungskombinationen bei neuen Apfelsorten
}

\author{
Suitable pollination combinations of new apple varieties \\ Combinazioni di nuove varietá di melo per un'impollinazione idonea
}

\author{
Irene Höller ${ }^{1}$, Markus Kellerhals², Simone Bühlmann-Schütz², Martin Brüggenwirth³, Jef Vercammen, Ann Gomand ${ }^{4}$, Walter Guerra ${ }^{1}$ \\ ${ }^{1}$ Versuchszentrum Laimburg, Pfatten, Italien \\ ${ }^{2}$ Agroscope, Wädenswil, Schweiz \\ ${ }^{3}$ Esteburg Obstbauzentrum Jork, Jork, Deutschland \\ ${ }^{4}$ Pcfruit, Sint-Truiden (Kerkom), Belgien
}

\section{ABSTRACT}

The working group of apple and pear variety and rootstocks testing within the EUFRIN network (European Fruit Research Institutes Network) has been conducting pollination trials annually using a standardized method. Since 2002, the institutes Agroscope (Switzerland), Esteburg Obstbauzentrum Jork (Germany) and Pcfruit (Belgium) have been exchanging their results with the Laimburg Research Centre (Italy). A comparison of all data makes it possible to define "suitable" and "unsuitable" pollen donors for new apple varieties such as MC 38 Crimson Snow ${ }^{\circledR}$, Nicoter Kanzi ${ }^{\circledR}$, Scifresh Jazz $^{\circledR}$ or Scilate Envy ${ }^{\circledR}$. Pollination success is determined by evaluating the fruit set, while the number of seeds provides information about the quality of pollination. An overlapping blooming period is fundamental for optimal pollination under natural conditions, therefore the respective flowering times are integrated in the pollination chart.

\section{KEYWORDS}

pollination, Eufrin, fruit set, number of seeds, flowering time

\section{CITE ARTICLE AS}

Höller Irene, Kellerhals Markus, Bühlmann-Schütz Simone et.al. (2020). Suitable pollination combinations of new apple varieties.

Laimburg Journal 02/2020

DOI: $10.23796 / \mathrm{LJ} / 2020.007$

\section{CORRESPONDING AUTHOR} Irene Höller

Laimburg 6, Pfatten, 39040 Auer (BZ), Italien

irene.hoeller@laimburg.it +390471969677 


\section{EINLEITUNG}

Apfelsorten sind weitgehend selbststeril und somit auf Fremdbefruchtung durch Pollen einer anderen geeigneten Sorte angewiesen. Weiters spielen für den finalen Fruchtansatz neben der primären Blütenknospenqualität vor allem Faktoren während der Blüte, wie Blühzeitpunkt, Aktivität der Bestäuberinsekten und Witterung (Temperatur, Niederschlag und Wind) eine entscheidende Rolle. Nach der Blüte ist die Samenanzahl pro Frucht, der Ernährungszustand der Bäume, das Wachstum und die Behangstärke für die Qualität der sich entwickelnden Früchte von großer Bedeutung.

\section{GENETIK}

Die meisten Apfelsorten sind diploid, haben also einen zweifachen Chromosomensatz und sind gute Pollenbildner. Bestimmte Apfelsorten sind triploid, das heißt, dass sie einen dreifachen Chromosomensatz aufweisen. Dazu zählen beispielsweise Boskoop, Gravensteiner und Jonagold. Eine Eigenheit dieser Sorten ist die Entwicklung von schlecht keimfähigem, nicht befruchtungsfähigem Pollen. Aufgrund dieser Pollensterilität scheiden solche Sorten als Pollenspender aus. Bei triploiden Sorten als Pollenempfänger sind genügend zeitgleich blühende, diploide Befruchter für die erfolgreiche Befruchtung erforderlich. Manche Sorten können sich aufgrund ihrer ähnlichen Genetik gegenseitig nicht befruchten. Sorten mit identischen S-Allelen gelten als nicht kompatibel. Diese genetische Inkompatibilität wird als Intersterilität bezeichnet und kann mit molekularen Methoden nachgewiesen werden. Sorten mit einem einzigen identischen S-Allel sind semi-kompatibel und können demnach nur mit rund $50 \%$ des Pollens befruchten. Dies reicht bei künstlichen Bestäubungen, z.B. im Rahmen von gezielten Kreuzungen, für einen ausreichenden Ertrag aus, denn dabei wird mit hohem Pollenüberschuss gearbeitet. Bei natürlichen Bestäubungen über Bienen hingegen ist davon auszugehen, dass Semi-Kompatibilität sehr wohl ein Thema ist. Diese Erkenntnisse wurden in einer am Versuchszentrum Laimburg durchgeführten Studie bestätigt [1], indem molekulargenetische Ergebnisse mit Befruchtungsdaten aus insgesamt 39 Jahren verglichen wurden. Sorten mit unterschiedlichen S-Allelen sind in der Regel kompatibel. Für Süßkirschen gilt dieser Zusammenhang als gesichert. Hier reicht die genetische Bestimmung der S-Allele aus, um den Befruchtungserfolg zusammen mit dem
Blühtermin vorherzusagen. Bei Äpfeln scheint dies laut Hanke M.V. (2017) [2] aber deutlich schwieriger zu sein. Zur Kompatibilität müssen u.a. sogenannte Anti-S-Allele vorhanden sein. Daher waren und sind beim Apfel Bestäubungsversuche im Freiland notwendig, um praktische Hinweise geben zu können.

\section{BLÜHZEITPUNKT}

Die Befruchtung in einer Obstanlage ist nur gesichert, wenn sich die Blütezeiten der entsprechenden Sorten ausreichend überschneiden. Im Idealfall sollte die Pollenspendersorte ein bis zwei Tage vor der Hauptsorte zu blühen beginnen [3]. Um den Blühbeginn der Sorten zu definieren, werden die in den Versuchsparzellen des Versuchszentrums Laimburg (220 m ü. NN) durchgeführten langjährigen phänologischen Beobachtungen herangezogen. Bei Sorten, welche nicht am Standort Laimburg vertreten sind, konnte entweder kein Blühtermin ermittelt werden oder es wurden Angaben aus der Literatur übernommen. Es ergibt sich eine Einteilung in fünf Klassen, nach dem Vorbild von Markus Kellerhals [4]: früh, mittelfrüh, mittel, mittelspät und spät. Um eine gegenseitige Befruchtung zu optimieren, sollten Pollenspender und Pollenempfänger in der Blütezeit nicht mehr als zwei Klassen voneinander abweichen.

\section{ARBEITSGRUPPE EUFRIN}

Geeignete Sortenkombinationen für eine optimale Befruchtung lassen sich anhand kontrollierter Kreuzungen ermitteln. Innerhalb der europäischen EUFRIN (European Fruit Research Institutes Network) Arbeitsgruppe der Sorten- und Unterlagenprüfer Apfel und Birne werden seit 2002 Informationen zu Bestäubungsversuchen ausgetauscht. Daran beteiligen sich neben dem Versuchszentrum Laimburg die Schweizer Forschungsanstalt Agroscope, das Obstbauzentrum Jork in Deutschland und das Versuchszentrum für Obstbau pcfruit in Belgien (Tab. 1). In den Jahren vorher erfolgte der Informationsaustausch in einer "Pollination Working Group", welche von den Versuchsstationen in Wilhelminadorp und später in Randwjik (NL) koordiniert wurden. Diese Arbeitsgruppe hat die Richtlinien für die Durchführung der Versuche [5] entwickelt, welche im Eufrin-Netzwerk mit kleineren Abweichungen bis heute berücksichtigt werden.

\section{KONTROLLIERTE BESTÄUBUNG}

Bei der kontrollierten Bestäubung werden die Blüten der Muttersorten im Ballonstadium mittels spezieller Baumwollsäcke eingehüllt, um eine Fremdbestäubung durch äußere Einflüsse zu verhindern. Dafür werden mindestens drei mehrjährige Äste von Bäumen ab dem dritten Standjahr ausgewählt, welche einen im Verhältnis zum Baumvolumen mittleren Blütenbesatz aufweisen. Sind die Blüten, geschützt durch die Baumwollsäcke aufgeblüht, erfolgt die Bestäubung mit dem getrockneten Pollen der Vatersorte, welcher manuell auf die Blüten der Muttersorte übertragen wird (Abb. 1). Der Fruchtansatz wird aus der prozentuellen Anzahl entwickelter Früchte nach dem Junifruchtfall im Verhältnis zu den bestäubten Blüten errechnet. Die durchschnittliche Anzahl der Samen pro Frucht wird nach der Ernte erhoben.

\section{FRUCHTANSATZ}

Über den Fruchtansatz kann der Erfolg der Bestäubungskombination untersucht werden. Der Befruchtungserfolg wird wie folgt eingestuft: Ein Fruchtansatz von 0 bis 5,9\% gilt als „schlecht“, jener von 6,0-9,9\% als mäßig und jener von über $10 \%$ als "gut“. Die Wiederholung ähnlicher Ergebnisse muss über mindestens zwei Jahre erfolgen, um die Eignung eines Pollenspenders für eine bestimmte Sorte zu definieren. Gibt es nur einjährige oder widersprüchliche Ergebnisse müssen noch weitere Bestäubungsversuche durchgeführt werden, bis die Eignung geklärt ist. In der Regel wurden bei Wiederholungen gleicher Bestäubungskombinationen an mehreren Jahren oder zwischen unterschiedlichen Standorten innerhalb der Arbeitsgruppe Eufrin vergleichbare Ergebnisse erzielt. Falls die Sorte im Bestäubungsversuch unter der Variante "frei abgeblüht", das heißt bei natürlichen Bedingungen ohne Einhüllung der Blüten eine „schlechte“ Eignung erhält, sind die "schlechten“ Bestäubungsergebnisse mit anderen Sorten ungültig. In diesem Fall könnte die Bestäubung beispielsweise aufgrund ungünstiger Wetterbedingungen nicht funktioniert haben. Zur Kontrolle der Selbststerilität wird bei Bestäubungsversuchen häufig der Fruchtansatz kontrolliert, der ohne jegliche Fremdbestäubung zustande kommt. Bei $80 \%$ aller Bestäubungskombinationen zur Kontrolle der Selbststerilität konnte durch Selbstbefruchtung kein zufriedenstellender Fruchtansatz erzielt werden. Bei einzelnen Sorten wie beispielsweise bei Delblush Tentation ${ }^{\circledR}$ oder 
Shinano Gold yello ${ }^{\circledR}$ wurde eine gewisse Neigung zur Selbstfruchtbarkeit festgestellt.

\section{ERGEBNISSE}

\section{WAHL POLLENSPENDER}

Aus den Ergebnissen von rund 850 Bestäubungsversuchen wurde die Eignung diverser Pollenspender für insgesamt 66 Sorten zusammengefasst (Tab. 2). Da in Praxisanlagen bei schorfresistenten Sorten im Idealfall ein resistenter Pollenspender gewählt wird, enthält die Tabelle die Information zur Schorfresistenz (Spalte Vf/Riv6). Jene Pollenspender, deren Nummern in der Tabelle mit dem Symbol "," abgebildet sind, eignen sich aufgrund der manuellen Bestäubungsversuche gut für die entsprechenden Sorten. Die Angabe der Blütezeit muss allerdings berücksichtigt werden, um unter natürlichen Bedingungen eine ausreichende Befruchtung zu erreichen. Die Sortennummern mit dem Symbol „,-" weisen auf unzufriedenstellende Bestäubungsergebnisse hin. Bei einer Reihe von Sorten bedarf es zur vollständigen Abklärung ihrer Eignung noch zusätzlicher Erfahrungen (ohne Symbol).

\section{SAMENANZAHL}

Die Anzahl der ausgebildeten Samen hängt maßgeblich von der Qualität der Befruchtung ab. Die Samenanzahl wurde bei insgesamt 769 Bestäubungsversuchen parallel zum Fruchtansatz erhoben. Abbildung 2 zeigt, dass bei $75 \%$ der Bestäubungsversuche ein durchschnittlicher Fruchtansatz von fast $30 \%$ erreicht wurde. $15 \%$ der bestäubten Sorten setzten aufgrund eines schlechten Befruchtungserfolgs im Mittel weniger als 3\% Früchte an. Bei schlechtem Fruchtansatz sind signifikant weniger Kerne vorhanden. Eine niedrige Samenanzahl kann den Junifruchtfall verstärken oder zu asymmetrischen Früchten führen [6]. Bei mäßigem und gutem Fruchtansatz ist die mittlere Samenanzahl mit 5,8 versus 5,9 Samen pro Apfel nahezu identisch.

\section{DISKUSSION}

Phänotypische Untersuchungen zum Befruchtungsverhalten verschiedener Apfelsorten liefern Erkenntnisse sowohl für die Forschung als auch für die Praxis. Wissenschaftlicher Nutzen kommt den Sortenzüchtungsprogrammen zugute, zum Beispiel, wenn es um die Auswahl geeigneter Kreuzungspartner geht.

\section{ZUSAMMENFASSUNG}

Die Arbeitsgruppe der Sorten- und Unterlagenprüfer Apfel und Birne aus dem EUFRIN Netzwerk (European Fruit Research Institutes Network) führt jährlich Bestäubungsversuche unter Anwendung einer standardisierten Methode durch. Seit dem Jahr 2002 tauschen neben dem Versuchszentrum Laimburg (Italien) die Institute Agroscope (Schweiz), Esteburg Obstbauzentrum Jork (Deutschland) und Pcfruit (Belgien) ihre Ergebnisse aus. Eine Zusammenfassung aller Daten ermöglicht es, für eine Reihe von neuen Apfelsorten wie beispielsweise MC 38 Crimson Snow ${ }^{\circledR}$, Nicoter Kanzi ${ }^{\circledR}$, Scifresh Jazz ${ }^{\circledR}$ oder Scilate Envy ${ }^{\circledast}$ "geeignete" und "ungeeignete" Pollenspender zu definieren. Der Bestäubungserfolg wird über die Ermittlung des Fruchtansatzes erhoben. Parallel dazu gibt die Samenanzahl Aufschluss über die Qualität der Bestäubung. Eine sich möglichst überlappende Blüte ist unter natürlichen Bedingungen Vorraussetzung für eine optimale Befruchtung, weshalb die jeweiligen Blütezeiten in den Tabellen der Pollenspender integriert sind.

\section{RIASSUNTO}

Il gruppo di lavoro sulle prove varietali e dei portinnesti di melo e pero dalla rete EUFRIN (European Fruit Research Institutes Network) esegue annualmente prove di impollinazione utilizzando un metodo standardizzato. Dal 2002 gli istituti Agroscope (Svizzera), Esteburg Obstbauzentrum Jork (Germania) e Pcfruit (Belgio) scambiano i loro risultati col Centro di Sperimentazione Laimburg (Italia). Tramite un raffronto di tutti i dati si possono definire impollinatori "adatti" e "non idonei" per una serie di nuove varietà di melo come $M C 38$ Crimson Snow ${ }^{\circledR}$, Nicoter Kanzi ${ }^{\circledR}$, Scifresh Jazz $^{\circledR}$ o Scilate Envy ${ }^{\circledR}$. II successo dell'impollinazione si valuta col grado dell'allegagione. II numero di semi fornisce invece informazioni sulla qualità dell'impollinazione. Una fioritura sovrapposta il più possibile temporalmente è un prerequisito per una fecondazione ottimale in condizioni naturali, motivo per cui le rispettive epoche di fioritura sono integrate nelle tabelle degli impollinatori.

\section{BEDEUTUNG S-ALLELE}

Die Daten der Bestäubungsversuche der Arbeitsgruppe Eufrin zeigen eine vergleichbare Samenanzahl bei mäßigem und gutem Fruchtansatz auf. Diese Ergebnisse weisen darauf hin, dass ein Überschuss an Pollen das Defizit an fruchtbaren Pollen bei semikompatiblen Bestäubungskombinationen ausgleichen kann [7]. Auch Mair (2018) [1] konnte bei gezielten Kreuzungsbestäubungen mit Pollenüberschuss keine statistischen Unterschiede in der Samenanzahl der Äpfel aus völlig kompatiblen und semi-kompatiblen Kreuzungspartnern feststellen. Bei inkompatiblen Sorten bzw. Sorten mit identischen S-Allelen wurde allerdings ein signifikant schlechterer Befruchtungserfolg beobachtet.

\section{OPTIMALE BEFRUCHTUNG}

In der Praxis kann eine Bestäubung mit einem semi-kompatiblen Partner unter Umständen problematisch sein, vor allem wenn bestimmte Faktoren den Befruchtungsvorgang erschweren. Das Wachstum des Pollenschlauches im Griffel benötigt bei $8^{\circ} \mathrm{C}$ z.B 9 Tage und bei $15^{\circ} \mathrm{C}$ nur 2 Tage. Bei Temperaturen unter $5{ }^{\circ} \mathrm{C}$ wird das Pollenschlauchwachstum eingestellt [8]. Eine Bestäubung beim Aufblühen und kurz danach garantiert den höchsten und sichersten Fruchtansatz. Je besser die Bedingungen für eine optimale Befruchtung sind, desto mehr Samen werden gebildet. Eine höhere Samenanzahl begünstigt den Kalzium-Gehalt der Früchte [9] [10] und die Fruchtgröße [11] [12] [13]. Eine hohe Samenanzahl in der Frucht kann bei bestimmten Sorten zu einer Hemmung der 
Blütenknospeninduktion für das folgende Jahr führen, wenn der Behang sehr hoch ist.

Die Effizienz eines geeigneten Pollenspenders ist neben einer regelmäßigen Wiederblüte vom Blühzeitpunkt der Sortenkombinationen abhängig. Dieser kann je nach Bodeneigenschaften, klimatischen Bedingungen, Standjahr des Baumes und Unterlagenwahl variieren. Für die Definition der Blütezeit der entsprechenden Sorten haben sich phänologische Aufzeichnungen an ein und demselben Standort über mehrere Jahre bewährt.

\section{VORTEILE ZIERAPFELSORTEN}

Nach Mantinger (2000) [8] sollen in einer Apfelanlage zwischen 5-10\% Befruchtersorten gut verteilt eingestreut sein. Im Südtiroler Obstbau sind die meisten Parzellen relativ klein strukturiert, weshalb in diesen Anlagen selten Befruchtersorten oder -reihen integriert werden. Bei größeren Flächen mit
Sorten, die sich gegenseitig nicht optimal befruchten können, sind Zieräpfel als Pollenspender oft die erste Wahl. Zierapfelsorten zeichnen sich meist durch Krankheitsresistenzen und einer reichlichen Blüte aus. Sie zeigen kaum Alternanz, wenn sie nach der Blüte geschnitten werden [11]. Vorteilhaft sind das Entfallen eines zusätzlichen Pflückgangs und der schwache Wuchscharakter, welche die Pflege verringern. Golden Hornet und Prof. Sprenger sind schorfresistent, während Evereste und Golden Gem noch zusätzlich eine Feuerbrandresistenz haben [4]. Golden Hornet ist allerdings sehr blutlausanfällig und neigt zur verstärkten Ausbildung von Fruchtmumien, die als Inokulum für Pilzkrankheiten dienen. Zierapfelsorten tragen oft das Allel S26, das bei keinem Kulturapfel vorhanden ist und daher auf eine gute Bestäubungsleistung schließen lässt.

Da in den letzten Jahrzehnten die genetische Variabilität des Sortenspektrums im Anbau verhältnismäßig kleiner und der Verwandtschaftsgrad der Sorten enger geworden ist, kann der Einsatz von Pollenspendersorten durchaus eine Überlegung wert sein. Aufgrund von Pollensterilität, Intersterilität oder unterschiedlicher Blühzeiten sind allerdings nur gewisse Sorten miteinander kombinierbar.

\section{DANKSAGUNG}

An Reinhold Stainer, Karin Gummerer, Robert Stocker, Martin Höller, Edmund Ebner, Nadia Sanin und Gerold Frank für die Planung und Durchführung der Bestäubungsexperimente am Versuchszentrum Laimburg.

An Rolf Stehr, Gesche Schacht, Julia Hauschildt, Rebecca Ohlig, Jennifer Kruse und Henning Harms für die Planung und Durchführung der Bestäubungsexperimente am Esteburg Obstbauzentrum Jork. 


\section{LITERATUR}

[1] Mair T., Höller I., Guerra W., Letschka T (2018). A molecular dating service: finding the perfect match for every apple. Acta Horticulturae (1203), 99-104,

DOI: 10.17660/ActaHortic.2018.1203.15.

[2] Hanke M.V., Flachowsky H. (2017). Obstzüchtung und wissenschaftliche Grundlagen. Springer, Berlin, Deutschland, hier pp. 4951.

[3] Tailleur C., Baab G., Lorenz J. (2018). Befruchtung optimieren - Fruchtbarkeit steigern. Öko-Obstbau 1, 7-12. Retrieved June 15, 2020, from https://www.foeko.de/wpcontent/uploads/2018/05/1-2018-kernobstbefruchtungsverhaeltnisse.pdf.

[4] Kellerhals M., Schütz S., Christen D. et al. (2014). Befruchtung der Obstsorten. Agroscope Transfer 41, 4-22. Retrieved June 15, 2020, from https://www.agroscope.admin.ch/agroscope/de/home/publikationen/suchen/agroscope-transfer/band-175.html.

[5] Wertheim S.J. (1996). Methods for cross pollination and flowering assessment and their interpretation. Acta Horticulturae 423, 237241, DOI: 10.17660/ActaHortic.1996.423.30.
[6] Sheffield C.S., Ngo H.T., Azzu N. (2016). A manual on apple pollination. Food and Agriculture Organization of the United Nations, Rome, Italy, hier pp. 8-9. Retreived June 15, 2020, from http://www.fao.org/mwg-internal/de5fs23hu73ds/progress?id=p/HH5uJgCF.

[7] Schneider D., Stern R.A., Goldway M. (2005). A comparison between semi- and fully compatible apple pollinators grown under suboptimal pollination conditions. HortScience 40 (5), 1280-1282, DOI: $10.21273 / \mathrm{HORTSCI} .40 .5 .1280$.

[8] Mantinger H. (2000). Hochwertige Fruchterträge durch optimale Befruchtung im Apfelanbau. Schweizer Zeitschrift für Obst- und Weinbau 136 (10), 209-212.

[9] Volz R.K., Tusk D.S., Ferguson I.B. (1996). Pollination effects on fruit mineral composition, seeds and cropping characteristics of 'Braeburn' apple trees. Scientia Horticulturae 66 (3-4), 169-180, DOI: $10.1016 / \mathrm{S} 0304-4238(96) 00934-X$.
[10] Sallato B. (2019). Calcium deficiencies in fruit. Good Fruit Grower 70 (7), 54-55. Retrieved June 15, 2020, from: https://www.goodfruit.com/sallato-calciumdeficiencies-in-fruit/.

[11] Botzner B. (1992). Zieräpfel - eine Alternative als Pollenspender? Obstbau Weinbau. Mitteilung des Südtiroler Beratungsringes 29 (5), 147-148.

[12] Buccheri M., Di Vaio C. (2004). Relationship Among Seed Number, Quality, and Calcium Content in Apple Fruits. Journal of Plant Nutrition 27 (10), 1735-1746,

DOI: $10.1081 /$ PLN-200026409.

[13] Vizzotto G., Driussi E., Pontoni M. et al. (2018). Effect of Flower Pollination on Fruit Set and Cropping in Apple. American Journal of Agriculture and Forestry 6 (5), 156-161, DOI: 10.11648/j.ajaf.20180605.16. 


\section{ANHANG 1: ABBILDUNGEN}

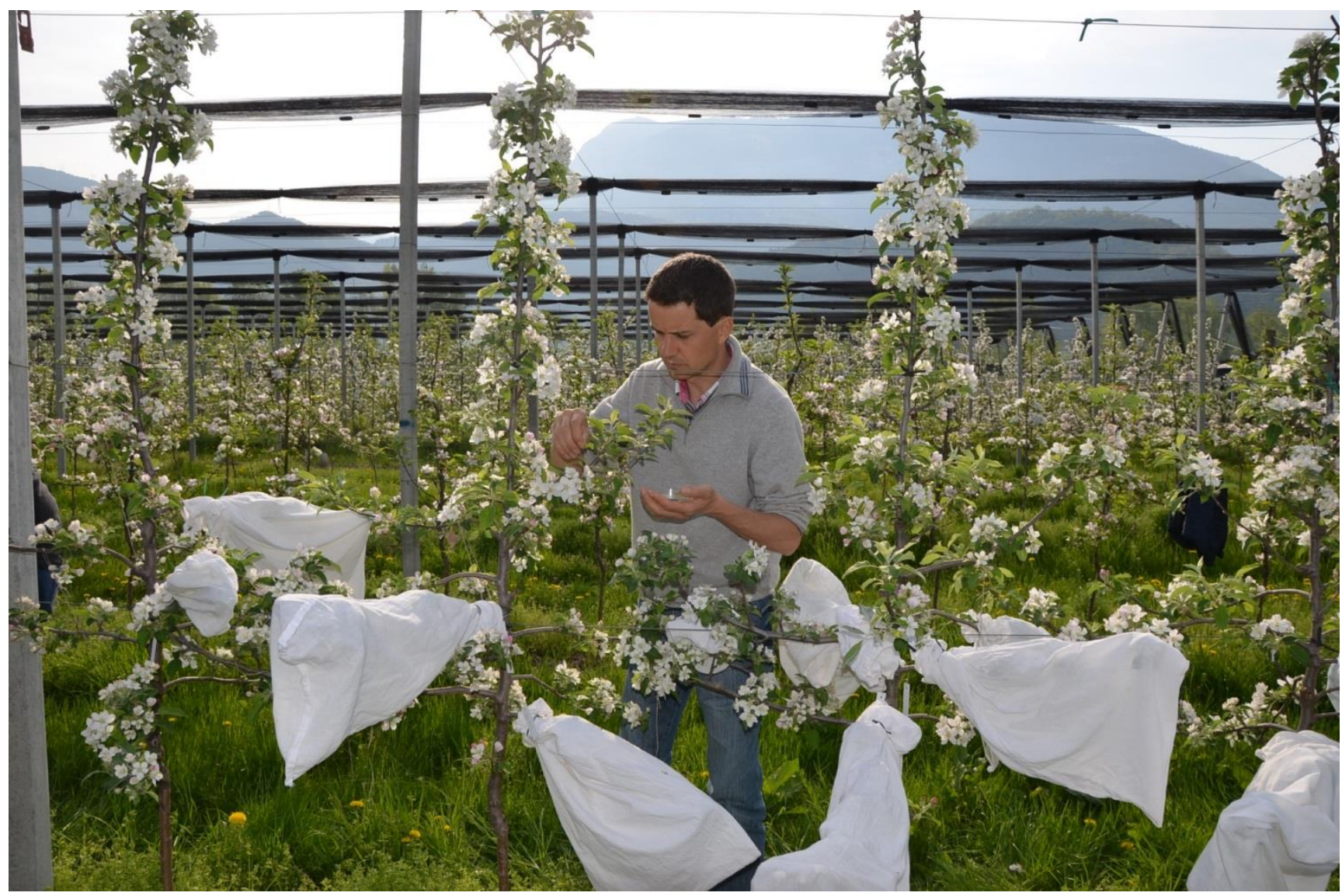

Abb. 1: Manuelle Bestäubung / Manual pollination.

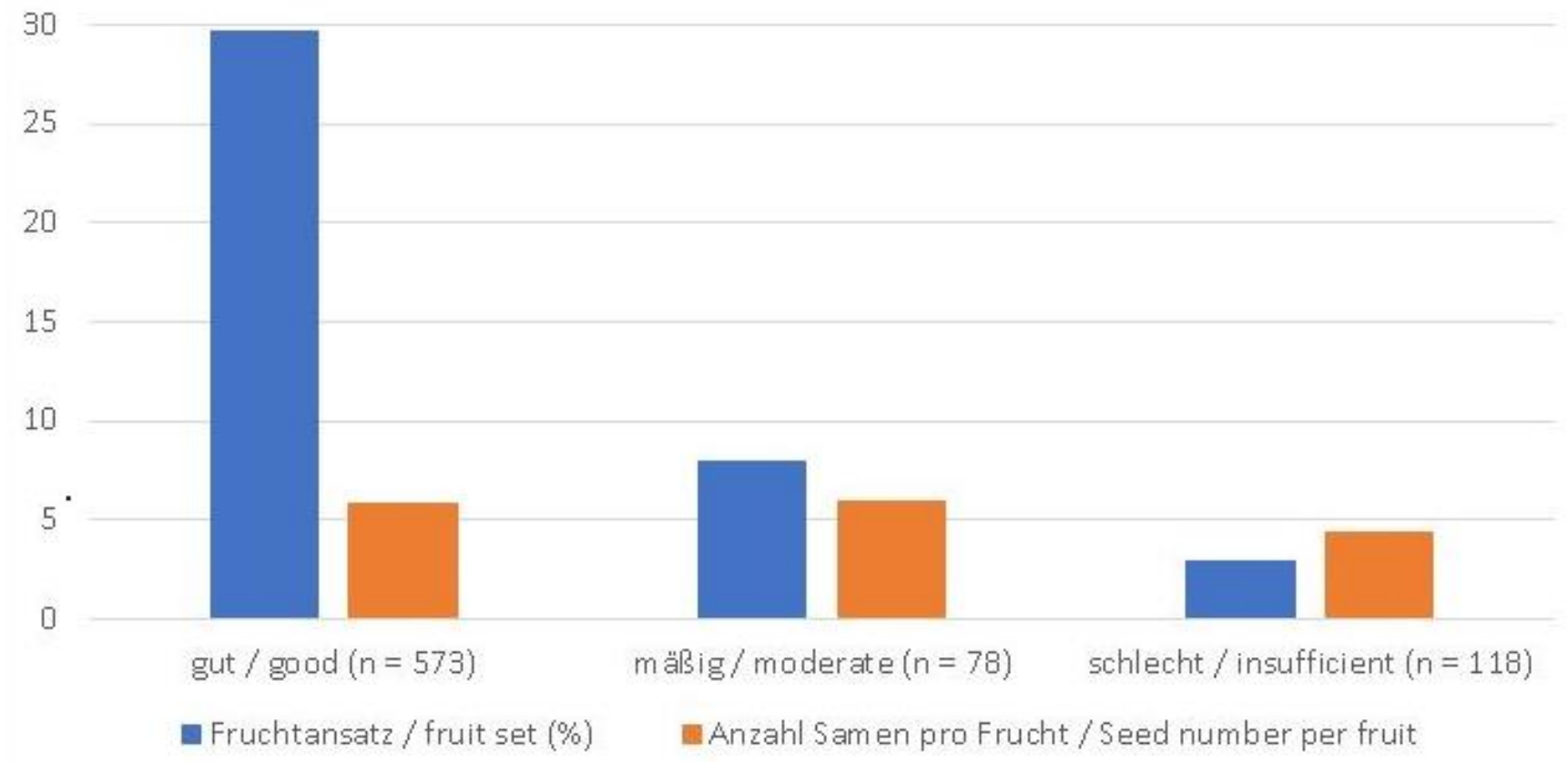

Abb. 2: Mittelwert aus Fruchtansatz und Anzahl Samen pro Frucht in Abhängigkeit vom Befruchtungserfolg, aus insgesamt 769 Bestäubungsversuchen der Arbeitsgruppe Eufrin // Mean value of fruit set and number seeds per fruit depending on pollination success, from a total of 769 pollination trials of Eufrin working group. 


\section{ANHANG 2: TABELLEN}

Tab. 1: Überblick Versuchsdaten nach Herkunft und Jahren // Overview trial data according to site and year.

\begin{tabular}{llc}
\multicolumn{1}{c}{$\begin{array}{c}\text { Herkunft } \\
\text { Site }\end{array}$} & $\begin{array}{l}\text { Jahre } \\
\text { Years }\end{array}$ & $\begin{array}{c}\text { Anzahl Bestäubungskombinationen } \\
\text { Number Pollination combinations }\end{array}$ \\
\hline Versuchszentrum Laimburg (I) & $2002-2019$ & 391 \\
\hline Forschungsanstalt Agroscope (CH) & $2002-2015$ & 106 \\
\hline Obstbauzentrum Jork (D) & 2002-2014 und 2016-2018 & 349 \\
\hline Obstbau pcfruit (BE) & 2013 und 2018 & 16
\end{tabular}




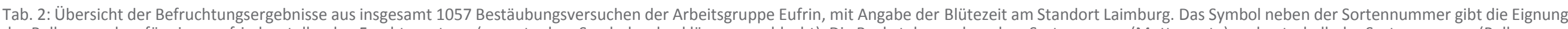

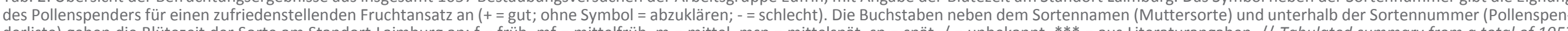

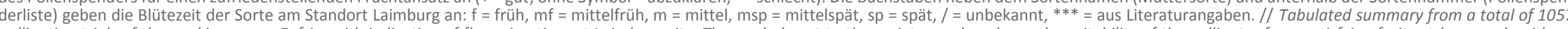

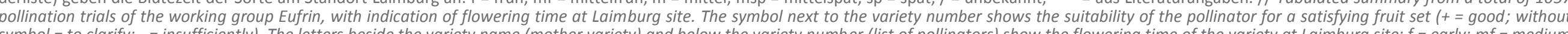

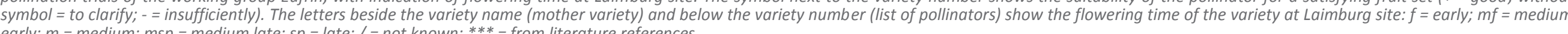
medium late $\cdot s p=$ late $/ 1=$ not known; $* * *=$ from literature references.

$\begin{array}{cccc}\text { Muttersorte } & \begin{array}{c}\text { Blütezeit } \\ \text { flowering } \\ \text { time }\end{array} & \text { Vf / } & \text { Piv6 } \\ \text { pollenspender } & \text { pollinator }\end{array}$

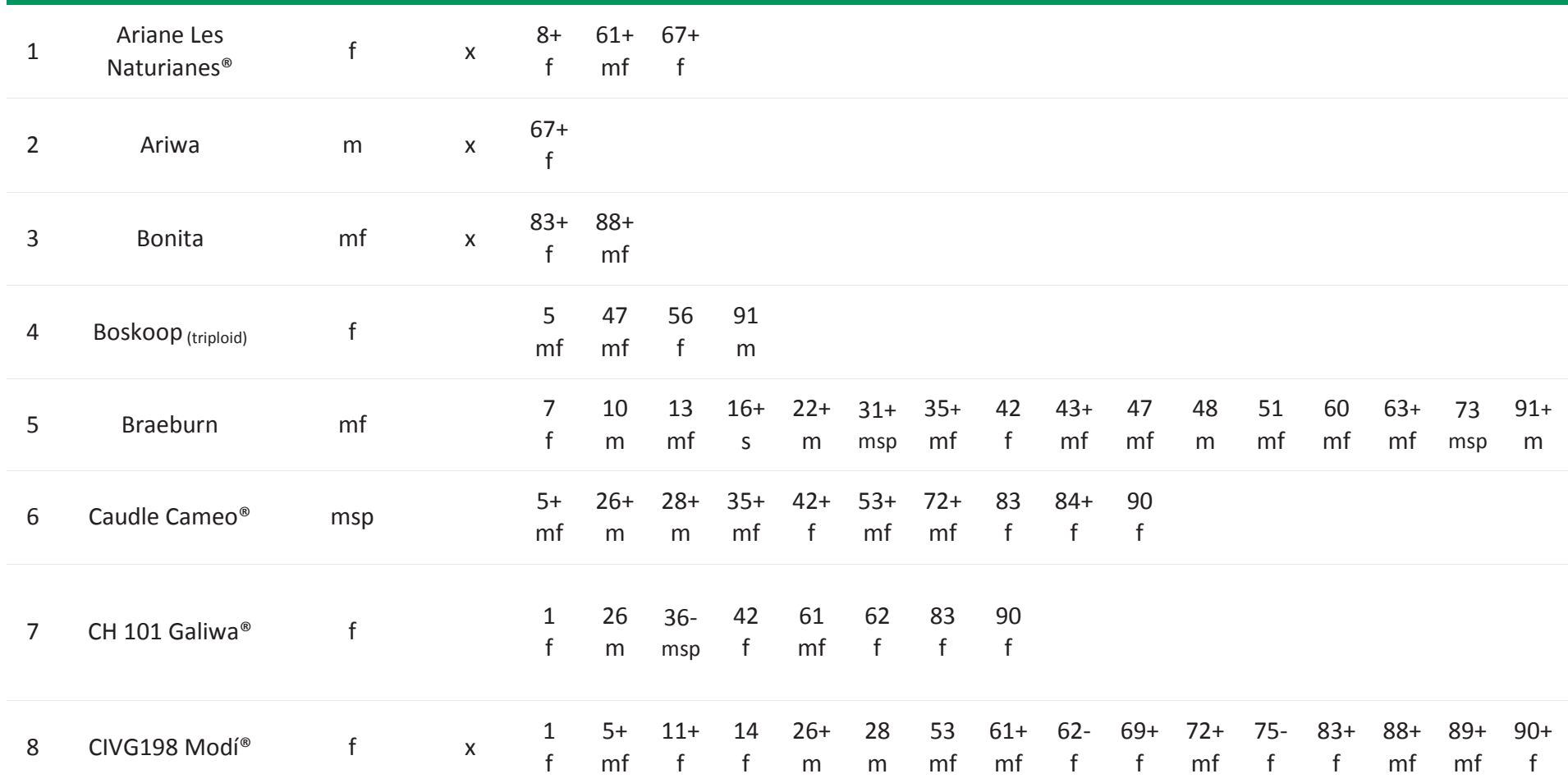




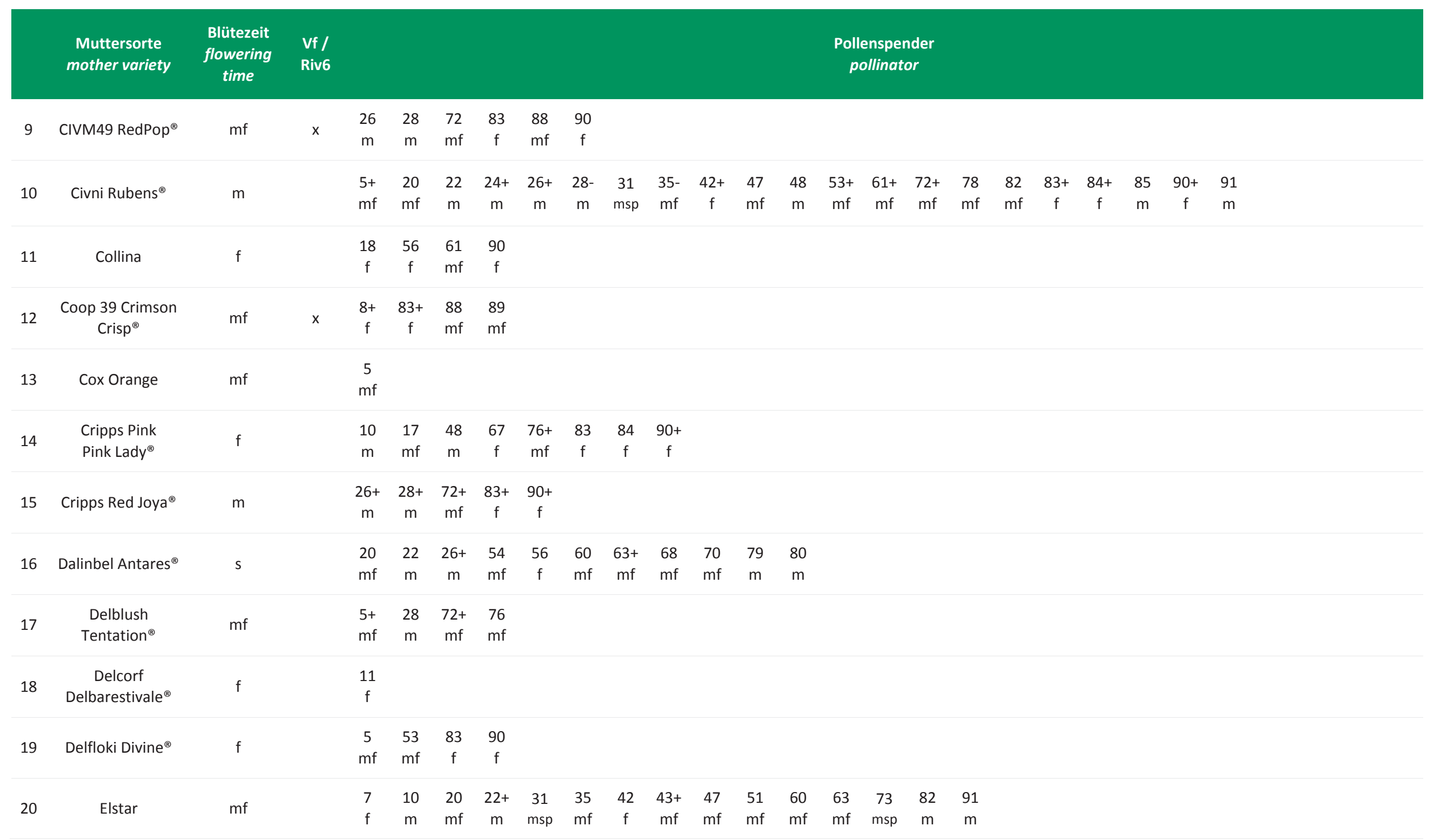




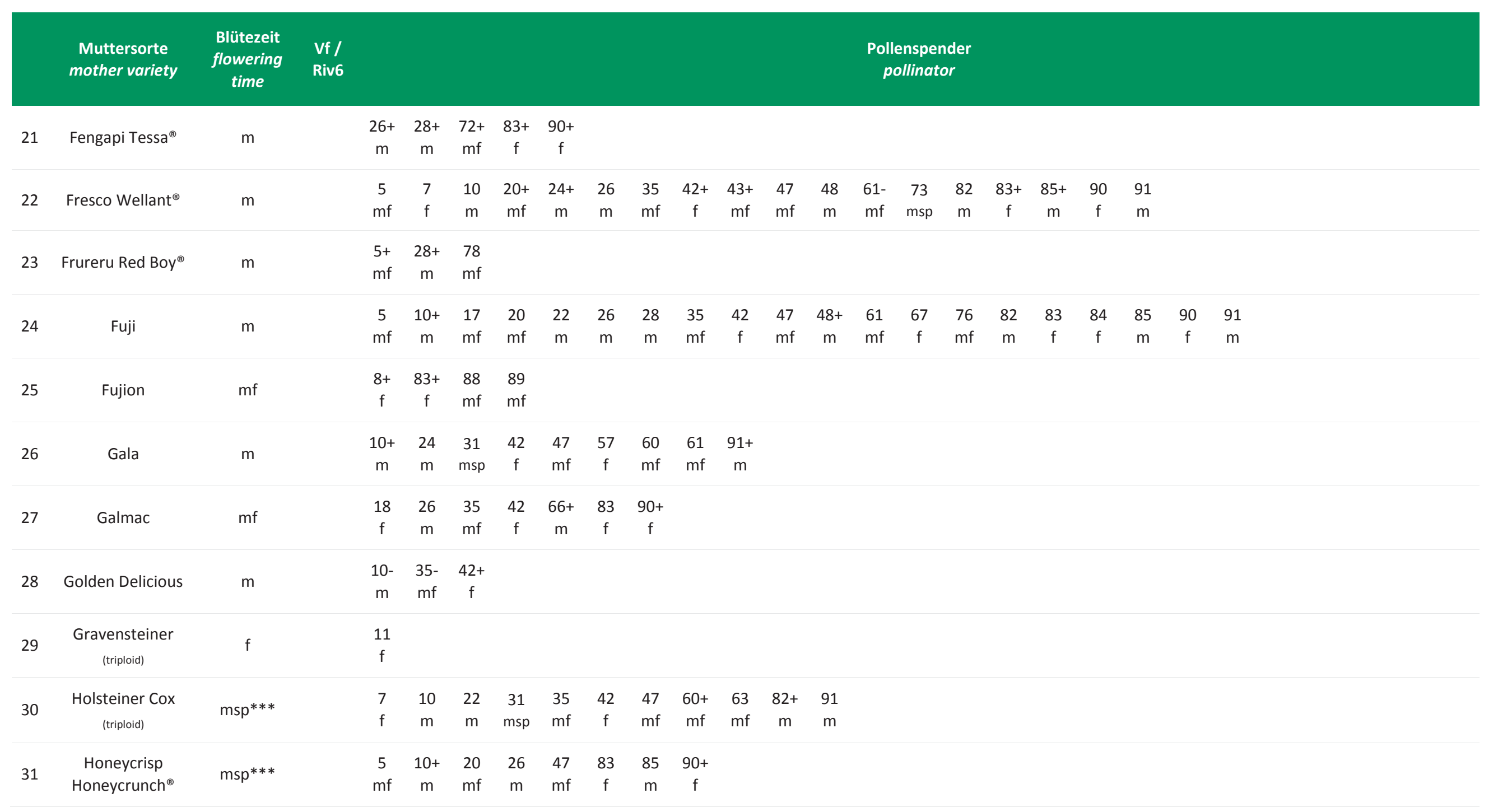




\begin{tabular}{|c|c|c|c|c|c|c|c|c|c|c|c|c|c|c|c|c|c|c|c|c|c|c|c|}
\hline & $\begin{array}{l}\text { Muttersorte } \\
\text { mother variety }\end{array}$ & $\begin{array}{c}\text { Blütezeit } \\
\text { flowering } \\
\text { time }\end{array}$ & $\begin{array}{l}\text { Vf / } \\
\text { Riv6 }\end{array}$ & \multicolumn{20}{|c|}{$\begin{array}{c}\text { Pollenspender } \\
\text { pollinator }\end{array}$} \\
\hline 32 & Ipador Giga ${ }^{\circledR}$ & $\mathrm{mf}$ & $x$ & $\begin{array}{l}26 \\
\mathrm{~m}\end{array}$ & $\begin{array}{l}28 \\
m\end{array}$ & $\begin{array}{l}72 \\
\mathrm{mf}\end{array}$ & $\begin{array}{c}83 \\
f\end{array}$ & $\begin{array}{l}88 \\
\mathrm{mf}\end{array}$ & $\begin{array}{c}90 \\
f\end{array}$ & & & & & & & & & & & & & & \\
\hline 33 & Jonagold (triploid) & $\mathrm{mf}$ & & $\begin{array}{l}7 \\
f\end{array}$ & $\begin{array}{l}10 \\
\mathrm{~m}\end{array}$ & $\begin{array}{c}16- \\
s\end{array}$ & $\begin{array}{l}22 \\
\mathrm{~m}\end{array}$ & $\begin{array}{c}31 \\
\mathrm{msp}\end{array}$ & $\begin{array}{l}35 \\
\mathrm{mf}\end{array}$ & $\begin{array}{c}42 \\
f\end{array}$ & $\begin{array}{l}43 \\
\mathrm{mf}\end{array}$ & $\begin{array}{l}47 \\
\mathrm{mf}\end{array}$ & $\begin{array}{l}60 \\
\mathrm{mf}\end{array}$ & $\begin{array}{c}63+ \\
\mathrm{mf}\end{array}$ & $\begin{array}{l}82 \\
m\end{array}$ & $\begin{array}{l}91 \\
\mathrm{~m}\end{array}$ & & & & & & & \\
\hline 34 & Kizuri Morgana ${ }^{\circledR}$ & $\mathrm{mf}$ & & $\begin{array}{c}44 \\
/\end{array}$ & $\begin{array}{l}72 \\
\mathrm{mf}\end{array}$ & $\begin{array}{c}84 \\
f\end{array}$ & $\begin{array}{l}85 \\
m\end{array}$ & $\begin{array}{l}91 \\
m\end{array}$ & & & & & & & & & & & & & & & \\
\hline 35 & $\begin{array}{l}\text { La Flamboyante } \\
\text { Mairac }^{\circledR}\end{array}$ & $\mathrm{mf}$ & & $\begin{array}{l}5+ \\
\mathrm{mf}\end{array}$ & $\begin{array}{c}10+ \\
\mathrm{m}\end{array}$ & $\begin{array}{l}20 \\
\mathrm{mf}\end{array}$ & $\begin{array}{l}22 \\
\mathrm{~m}\end{array}$ & $\begin{array}{l}24 \\
m\end{array}$ & $\begin{array}{c}26+ \\
m\end{array}$ & $\begin{array}{c}28- \\
m\end{array}$ & $\begin{array}{c}42 \\
f\end{array}$ & $\begin{array}{c}47+ \\
\mathrm{mf}\end{array}$ & $\begin{array}{l}48 \\
m\end{array}$ & $\begin{array}{l}53 \\
\mathrm{mf}\end{array}$ & $\begin{array}{l}61 \\
\mathrm{mf}\end{array}$ & $\begin{array}{c}72+ \\
\mathrm{mf}\end{array}$ & $\begin{array}{c}83+ \\
f\end{array}$ & $\begin{array}{c}84- \\
f\end{array}$ & $\begin{array}{l}85 \\
m\end{array}$ & $\begin{array}{c}90+ \\
f\end{array}$ & $\begin{array}{l}91 \\
\mathrm{~m}\end{array}$ & & \\
\hline 36 & Ladina & msp & & $\begin{array}{c}7+ \\
f\end{array}$ & $\begin{array}{l}60 \\
\mathrm{mf}\end{array}$ & $\begin{array}{c}61+ \\
\mathrm{mf}\end{array}$ & $\begin{array}{c}83 \\
f\end{array}$ & $\begin{array}{c}84 \\
f\end{array}$ & & & & & & & & & & & & & & & \\
\hline 37 & Lb 17906 & $f$ & $x$ & $\begin{array}{c}8+ \\
f\end{array}$ & $\begin{array}{c}83+ \\
f\end{array}$ & & & & & & & & & & & & & & & & & & \\
\hline 38 & Lb 4852 & $\mathrm{mf}$ & & $\begin{array}{c}26+ \\
m\end{array}$ & $\begin{array}{c}28+ \\
m\end{array}$ & $\begin{array}{c}72+ \\
\mathrm{mf}\end{array}$ & $\begin{array}{c}90+ \\
f\end{array}$ & & & & & & & & & & & & & & & & \\
\hline 39 & Maia1 EverCrisp ${ }^{\circledR}$ & s & & $\begin{array}{l}26 \\
m\end{array}$ & $\begin{array}{l}28 \\
m\end{array}$ & $\begin{array}{l}72 \\
\mathrm{mf}\end{array}$ & $\begin{array}{c}83 \\
f\end{array}$ & $\begin{array}{c}90 \\
f\end{array}$ & & & & & & & & & & & & & & & \\
\hline 40 & Mariella & $f$ & & $\begin{array}{c}26+ \\
m\end{array}$ & $\begin{array}{l}35 \\
\mathrm{mf}\end{array}$ & $\begin{array}{c}42+ \\
f\end{array}$ & & & & & & & & & & & & & & & & & \\
\hline 41 & $\begin{array}{c}\text { MC } 38 \text { Crimson } \\
\text { Snow }^{\circledR}\end{array}$ & $\mathrm{m}$ & & $\begin{array}{c}26+ \\
m\end{array}$ & $\begin{array}{c}28+ \\
m\end{array}$ & $\begin{array}{c}72+ \\
\mathrm{mf}\end{array}$ & $\begin{array}{c}83+ \\
f\end{array}$ & $\begin{array}{c}90+ \\
f\end{array}$ & & & & & & & & & & & & & & & \\
\hline 42 & Milwa Junami ${ }^{\circledR}$ & $f$ & & $\begin{array}{l}5+ \\
\mathrm{mf}\end{array}$ & $\begin{array}{l}10 \\
\mathrm{~m}\end{array}$ & $\begin{array}{l}20 \\
\mathrm{mf}\end{array}$ & $\begin{array}{l}22 \\
\mathrm{~m}\end{array}$ & $\begin{array}{c}24+ \\
m\end{array}$ & $\begin{array}{l}26- \\
m\end{array}$ & $\begin{array}{c}28+ \\
m\end{array}$ & $\begin{array}{l}35 \\
\mathrm{mf}\end{array}$ & $\begin{array}{c}40+ \\
f\end{array}$ & $\begin{array}{l}47+ \\
\mathrm{mf}\end{array}$ & $\begin{array}{l}48 \\
m\end{array}$ & $\begin{array}{c}53+ \\
\mathrm{mf}\end{array}$ & $\begin{array}{c}57 \\
f\end{array}$ & $\begin{array}{l}61 \\
\mathrm{mf}\end{array}$ & $\begin{array}{l}72+ \\
\mathrm{mf}\end{array}$ & $\begin{array}{l}82 \\
\mathrm{mf}\end{array}$ & $\begin{array}{c}83+ \\
f\end{array}$ & $\begin{array}{c}84+ \\
f\end{array}$ & $\begin{array}{cc}85 & 90+ \\
m & f\end{array}$ & $\begin{array}{l}91 \\
\mathrm{~m}\end{array}$ \\
\hline 43 & $\begin{array}{c}\text { Minneiska } \\
\text { Sweetango }\end{array}$ & $\mathrm{mf}$ & & $\begin{array}{c}5 \\
\mathrm{mf}\end{array}$ & $\begin{array}{l}20 \\
\mathrm{mf}\end{array}$ & $\begin{array}{l}22 \\
\mathrm{~m}\end{array}$ & $\begin{array}{l}26 \\
\mathrm{~m}\end{array}$ & $\begin{array}{l}28 \\
\mathrm{~m}\end{array}$ & $\begin{array}{l}43 \\
\mathrm{mf}\end{array}$ & $\begin{array}{l}47 \\
\mathrm{mf}\end{array}$ & $\begin{array}{l}72 \\
\mathrm{mf}\end{array}$ & $\begin{array}{c}83 \\
f\end{array}$ & $\begin{array}{l}85 \\
m\end{array}$ & $\begin{array}{l}87 \\
\mathrm{mf}\end{array}$ & $\begin{array}{c}90- \\
f\end{array}$ & & & & & & & & \\
\hline
\end{tabular}




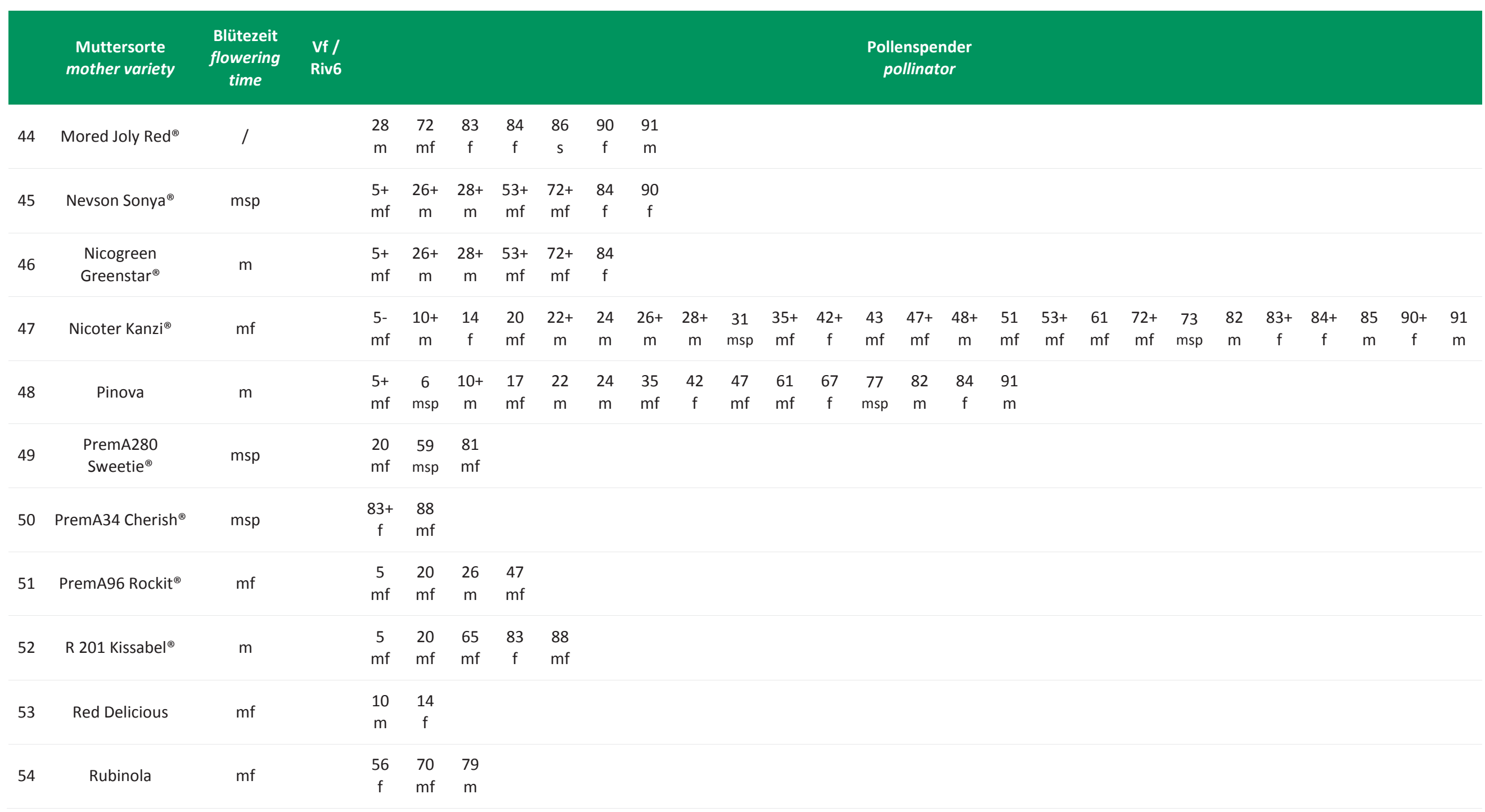




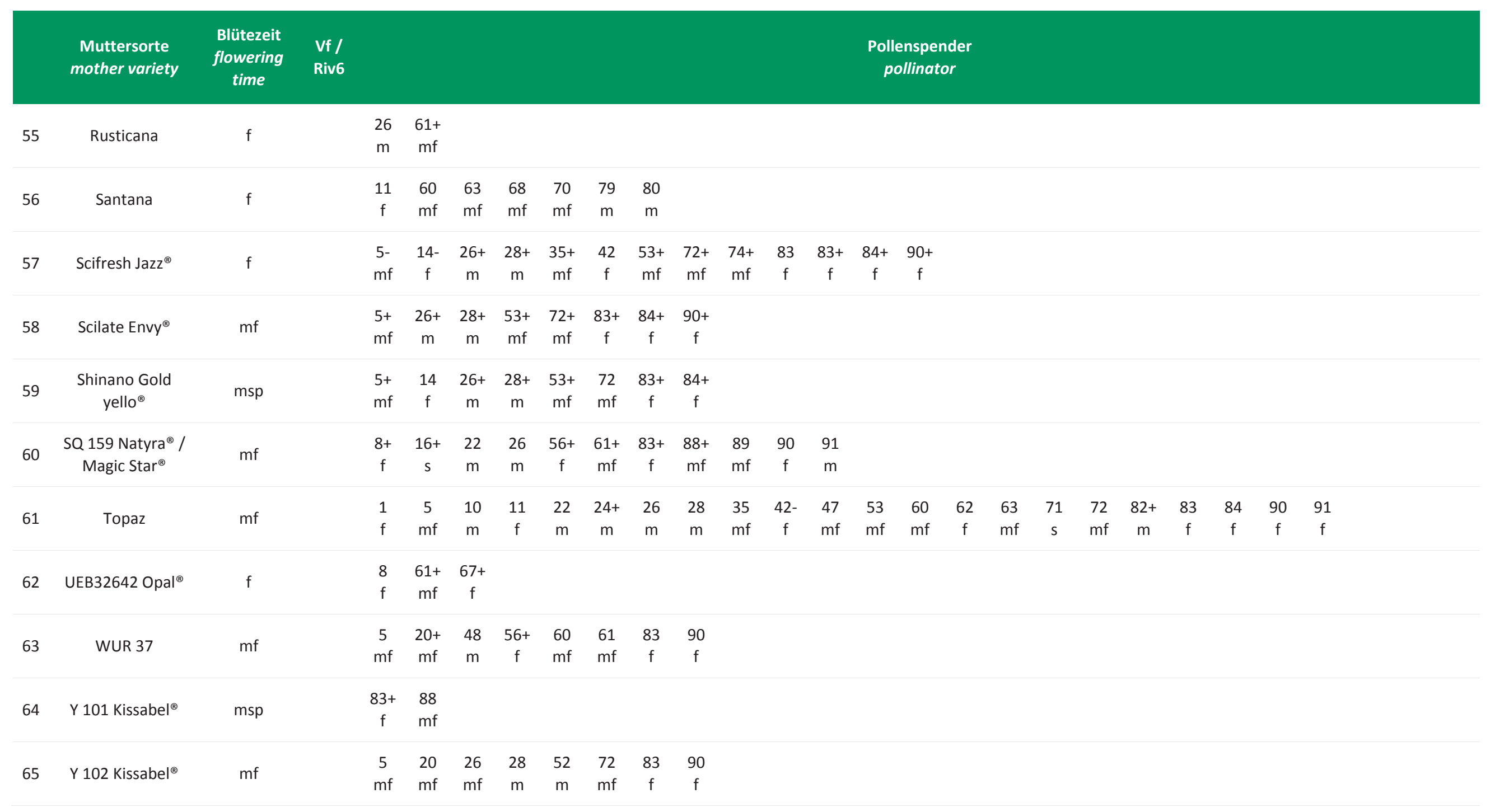




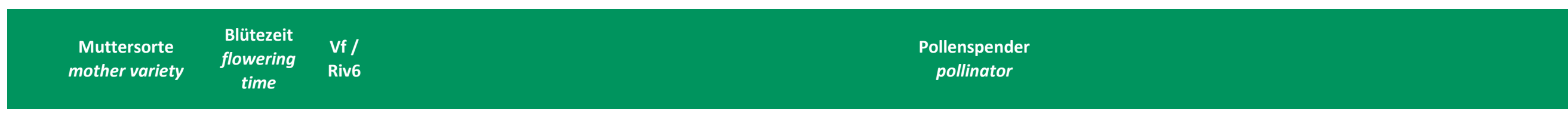

$\begin{array}{cccccccc}66 & & & 5 & 18- & 20 & 27+ & 47 \\ & & \mathrm{mf} & \mathrm{f} & \mathrm{mf} & \mathrm{mf} & \mathrm{mf}\end{array}$

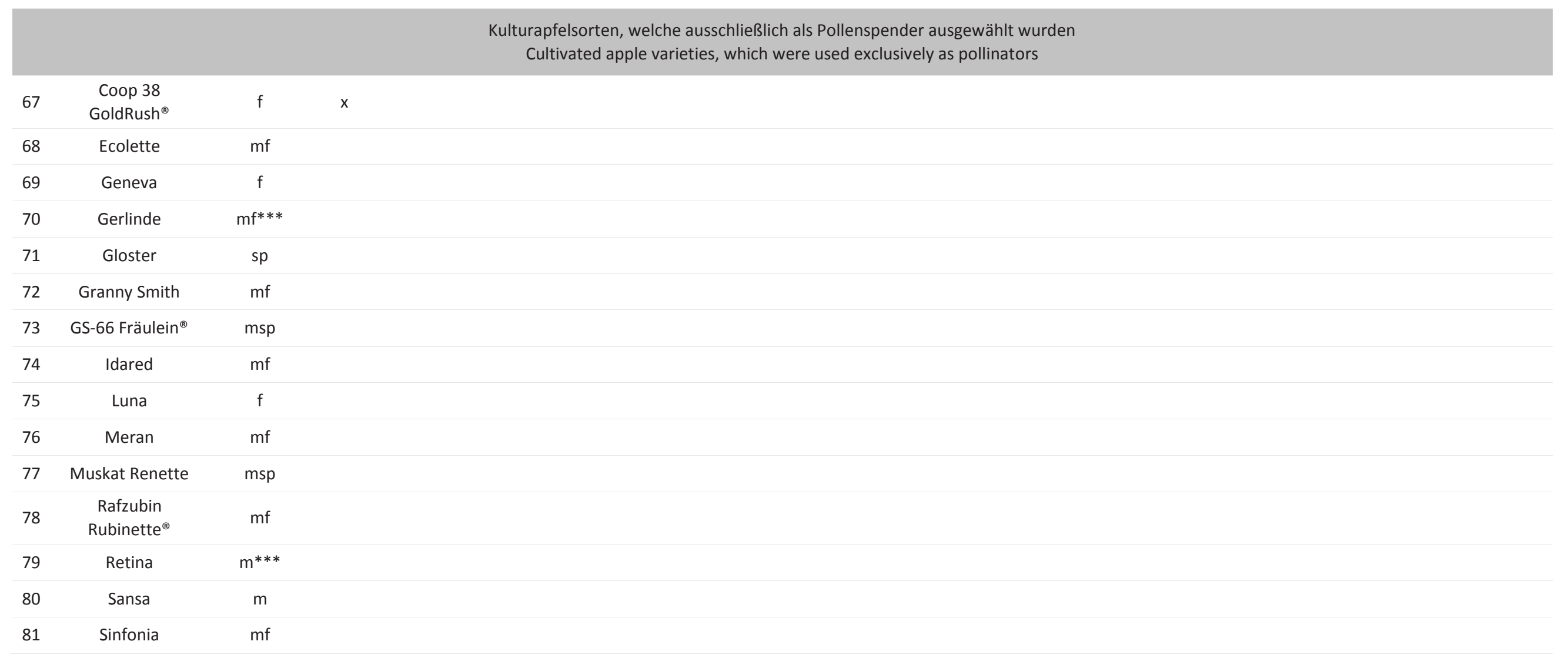




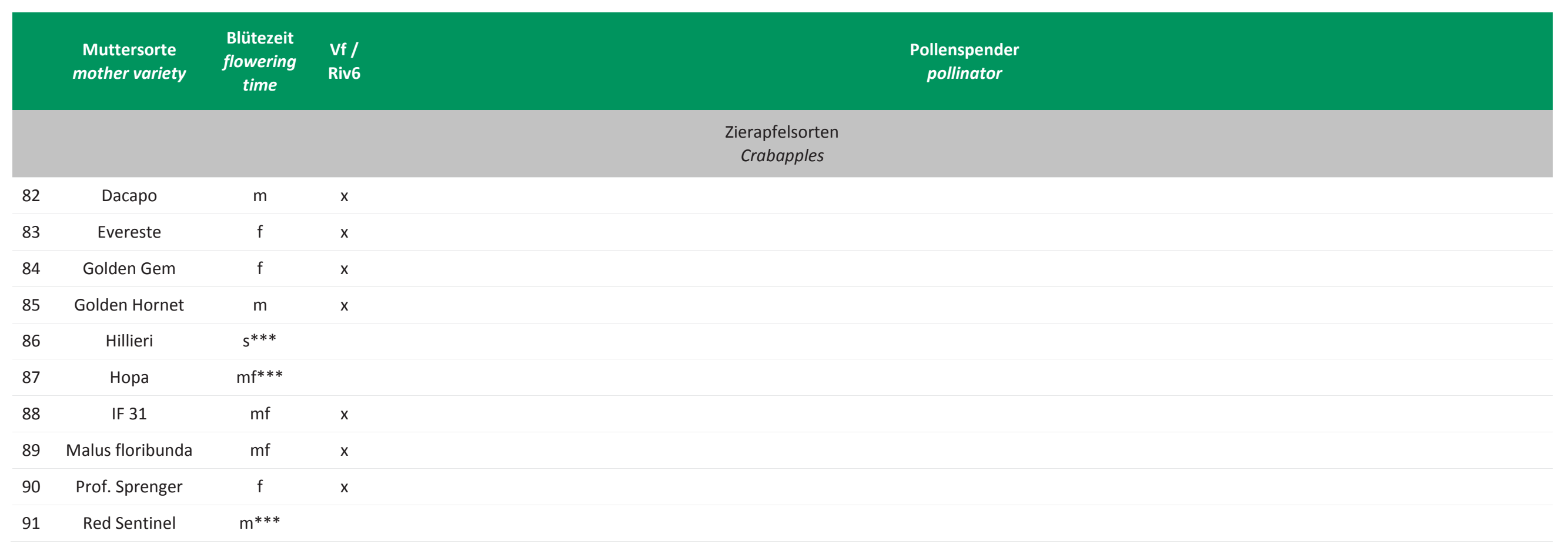

\section{(ब) $(1) \otimes$}

Dieses Werk ist lizenziert unter einer Creative Commons Namensnennung-Nicht kommerziell 4.0 International Lizenz.

Quest'opera è distribuita con Licenza Creative Commons Attribuzione - Non commerciale 4.0 Internazionale.

This work is licensed under a Creative Commons Attribution-NonCommercial 4.0 International License.

Für alle Abbildungen und Tabellen ohne Nennung des Urhebers gilt: @ Versuchszentrum Laimburg.

Per tutte le immagini e tabelle senza menzione dell'artefice vale: (C) Centro di Sperimentazione Laimburg.

For all figures and tables without mention of the originator applies: () Laimburg Research Centre. 